whether infection resulted from an epidemiological link between the cases.

The Canadian study collected data over 2 years (May-2015 to April-2017). Eight cases of laboratory-confirmed listeriosis were reported in newborns and infants. Six cases had EOD and 2 had LOD. All the six EOD cases presented as septic on DOL 1 (median: 0.5 hours, range: 0-2 hours). All EOD cases had bacteremia and one also meningitis. All EOD cases needed ICU (or NICU) admission; 2 of the EOD cases died. Of the LOD cases, one case became symptomatic on DOL 9 and the other presented on DOL 20 with seizures. Both LOD cases survived the episode, but in one case, the infant suffered permanent sequelae (hemiplegia).

Conclusion While the incidence of neonatal listeriosis recorded in Switzerland exceeded the incidence as could be expected from routine surveillance data, this increase likely related to the unusual cluster of cases. Contrary, the number of cases reported in Canada was far below the incidence as could be expected from the reported incidence of neonatal listeriosis in the literature (table 1). Under-reporting was unlikely as no additional listeriosis were reported through the national notifiable system. This apparent reduction in incidence of EOD could reflect perinatal antibiotic prophylaxis for Group B streptococcus; conclusions about LOD will require longer study period.

\begin{tabular}{lll} 
Abstract 19 Table 1 & & \\
\hline & Switzerland & Canada \\
\hline Study duration [months] & 8 & 24 \\
Number of cases & 4 & 8 \\
$\begin{array}{l}\text { Incidence/ } 100^{\prime} 000 \text { live births } \\
\text { Sex ratio (M:F) }\end{array}$ & 4.5 & 1 \\
Gestational age at birth [range of weeks & $1: 1$ & $1: 1.7$ \\
completed] & $34-39$ & $25-41$ \\
Birth weight [range in g] & $2220-3770$ & $880-$ \\
& & 3900 \\
Age of infant at onset of symptoms (range) & 17 hour - 8d & $0-20 \mathrm{~d}$ \\
Ratio of early- to late-onset disease & $1: 1$ & $3: 1$ \\
\hline
\end{tabular}

\section{ADHERENCE TO TREATMENT AND REDUCTION IN BMI SDS ASSOCIATED WITH LOWER HBA1C, 1 YEAR AFTER DIAGNOSIS OF TYPE 2 DIABETES IN YOUTH}

${ }^{1} \mathrm{TP}$ Candler, ${ }^{2,3} \mathrm{O}$ Mahmoud, ${ }^{4} \mathrm{RM}$ Lynn, ${ }^{1} \mathrm{AA}$ Majbar, ${ }^{5} \mathrm{TG}$ Barrett, ${ }^{1} \mathrm{JP}$ Hamilton-Shield. ${ }^{1}$ NIHR Biomedical Research Centre: Nutrition, Diet and Lifestyle theme, School of Oral and Dental Sciences, University of Bristol, Bristol, UK; ${ }^{2}$ Population Health Sciences, Bristol Medical School, University of Bristol, UK; ${ }^{3}$ Department of Applied Statistics, Helwan University, Egypt; ${ }^{4}$ British Paediatric Surveillance Unit, Royal College of Paediatrics and Child Health, UK; ${ }^{5}$ Institute of Cancer and Genomic Sciences, University of Birmingham, UK

\subsection{6/archdischild-2018-rcpch.478}

Objective To report outcomes from a national cohort of children and young people with type 2 diabetes (T2DM), 1 year post diagnosis

Research design and methods

1 year follow up of a cohort of children ( $<17$ years) with T2DM reported through the British Paediatric Surveillance Unit between April 2015 to April 2016. This established an overall UK incidence of 0.72 per 100000 per year (2.92/ 100,000 in Asians, 1.67/100,000 Black/African/Caribbean/Black British)
Results Ninety-nine (93\%) of the 106 notified cases had data available for one-year review. Of these, seven had been lost to follow up and one had the diagnosis revised. The mean age at follow up was 15.3 years. Of those with data, average BMI SDS was 2.72 with a mean increase of 0.14 SDS over a year (92\% remained overweight or obese). Only $\sim 15 \%$ of cases achieved a reduction in body weight of $5 \%$ or more from baseline. Median $\mathrm{HbA1c}$ was $53 \mathrm{mmol} / \mathrm{mol}$ (range 31$130 \mathrm{mmol} / \mathrm{mol}$ ) and $\sim 40 \%$ attained the UK national target of $<48 \mathrm{mmol} / \mathrm{mol}$. HbA1c was associated with BMI SDS change at 1 year $(p=0.007)$ and clinician reported compliance and attendance concerns $(p \leq 0.0001)$. In over half of cases, clinicians reported issues with compliance and attendance. Mean clinic attendance was 77\%. Metformin was the most frequently used agent in management at baseline (77\%) and follow up (87\%). Microalbuminuria at 1 year was seen in $16.4 \%$ of cases compared to $4.2 \%$ at baseline.

Conclusions Good compliance and BMI reduction is associated with better outcomes, one year after diagnosis in early onset T2DM. It is concerning that the prevalence of microalbuminuria increases four-fold in this short time frame.

\section{I11 21 YEARS OF SURVEILLANCE FOR VITAMIN K DEFICIENCY BLEEDING IN INFANTS: POLICY CHANGES IN AUSTRALIA AND INTERNATIONAL COMPARISONS}

\footnotetext{
${ }^{1,2}{ }^{2}$ A Zurynski, ${ }^{1} \mathrm{G}$ Ridley, ${ }^{3} \mathrm{~B}$ Jalaludin, ${ }^{1,2,4} \mathrm{E}$ Elliott, on behalf of the APSU VKDB Study Group. 'Australian Paediatric Surveillance Unit, Kids Research Institute, Westmead, Australia; ${ }^{2}$ Discipline of Paediatrics and Child Health, The University of Sydney, Australia; ${ }^{3}$ Centre for Research, Evidence Management and Surveillance, South Western Sydney and Health District; and the University of New South Wales, Australia; ${ }^{4}$ The Sydney Children's Hospitals Network, Westmead, Australia
}

\subsection{6/archdischild-2018-rcpch.479}

Background The NHMRC recommends Vitamin $\mathrm{K}$ at birth for the prevention of Vitamin K Deficiency Bleeding (VKDB). Intramuscular injection (IMI) is the preferred route of administration but oral dosing may be chosen. We describe the incidence and outcome of VKDB during changes in vitamin $\mathrm{K}$ prophylaxis policy and the introduction of a new formulation of Vitamin K.

Methods Infants with VKDB were identified by active surveillance through the Australian Paediatric Surveillance Unit (APSU), 1993-2012. Demographics, clinical characteristics and outcomes were collected via questionnaire completed by the reporting doctor.

Results Fifty-one cases of VKDB were reported; 36 late onset and 15 early/classical. Twenty-seven (53\%) infants received no vitamin $\mathrm{K}$, in $22(81 \%)$ of these parents refused consent, and this was the case in $92 \%$ of the 12 home births. The national incidence of VKDB was 0.9/100,000 live births per annum. Higher incidence was detected in $1993(4.6 / 100,000)$ when oral dosing was recommended and in $2000(2.3 / 100,000)$ coinciding with changes in Vitamin $\mathrm{K}$ formulation to enable oral dosing. Of 7 infants who received oral doses of Vitamin $\mathrm{K}$, only 1 received the complete course of 3 doses. Surprisingly, 6 cases of VKDB were reported in 2013 (2/100,000); parents refused consent in 5 .

Thirty-one (61\%) infants had a good outcome after bleeding was corrected with vitamin K. Ongoing morbidity was almost exclusively confined to those with late onset VKDB $(12 / 36,30 \%)$ and related to underlying liver disease, or intracranial haemorrhage. Six (12\%) infants died including one in 2013 due to intracranial 
haemorrhage. Adverse outcomes have also been reported in New Zealand and in the United Kingdom among predominantly breastfed infants whose parents refuse Vitamin K.

Conclusion IMI injection remains the most reliable and recommended mode of administering vitamin $\mathrm{K}$ to newborns, however, some parents refuse to consent to Vitamin $\mathrm{K}$ prophylaxis, even in recent years. A broad awareness and education campaign is needed to prevent infant deaths due to VKDB.

\section{USING A SURVEILLANCE METHODOLOGY TO ESTIMATE THE INCIDENCE OF TRANSITION FOR YOUNG PEOPLE WITH ATTENTION DEFICIT HYPERACTIVITY DISORDER (ADHD) REQUIRING ONGOING SUPPORT FROM CHILD TO ADULT SERVICES}

${ }^{1} \mathrm{H}$ Eke, 'A Janssens, ${ }^{2} \mathrm{RM}$ Lynn, 'T Ford. 'University of Exeter Medical School, Exeter, UK; ${ }^{2}$ British Paediatric Surveillance Unit

10.1136/archdischild-2018-rcpch.480

Aims The surveillance study is one stream of the ADHD in transition between children's services and adult services (CATCh-Us) study and is run in collaboration with the British Paediatric Surveillance Unit (BPSU) and the Child and Adolescent Psychiatry Surveillance System (CAPSS) across the United Kingdom. It aims to quantify the need for young people with ADHD to transition from children's to adult services, describe this group of young people, estimate the proportion that successfully transition and describe the proportion experiencing optimal transition.

Methods Starting in November 2015 for 13 months, paediatricians and psychiatrists registered with BPSU/CAPSS $(n=4500)$ reported monthly any patients seen with a diagnosis of ADHD, within 6 months of the age boundary of the service, requiring transition to an adult service to continue their ADHD treatment and supervision. All clinicians reporting a case received a questionnaire to confirm eligibility and to gather further information. A follow up questionnaire was sent nine months after notification of an eligible case to establish the details and outcome of the transition.

Results 228 eligible cases were reported via BPSU and 138 via CAPSS, with initial questionnaire response rates of $91 \%$ and $67 \%$ respectively. Follow up questionnaire response rates were $67 \%$ and $60 \%$ respectively. There were no duplicate cases reported across both systems. Preliminary results indicate poor transition processes with less than $25 \%$ of clinicians holding a transition planning meeting, only 25\% having a handover period and less than $50 \%$ having the referral to an adult service accepted; $50 \%$ were referred to a specialist adult ADHD service and $12 \%$ to primary care.

Conclusion Results indicate that patients requiring transition are not experiencing a smooth or successful process. The effectiveness of using a surveillance study methodology to estimate the incidence of transition is reported, as well as study findings. It is likely that results are an underestimate of the incidence of cases requiring transition to an adult service; it relies on clinicians reporting regularly and accurately, not all clinicians treating ADHD patients are registered with BPSU or CAPSS, and patients can be seen by other professionals not reporting via these systems.

Funding acknowledgement This project was funded by the National Institute for Health Research - Health Services and Delivery Research programme (project ref: 14/21/52)

\section{FEMALE GENITAL MUTILATION (FGM) SURVEILLANCE IN UNDER 16 YEARS OLDS IN THE UK AND IRELAND}

${ }^{1} \mathrm{~N}$ Ayadi O'Donnell, ${ }^{2} \mathrm{~K}$ Pall, ${ }^{2} \mathrm{M}$ Leoni, ${ }^{3} \mathrm{G}$ Debelle, ${ }^{4} \mathrm{RM}$ Lynn, ${ }^{5} \mathrm{~A}$ Armitage, ${ }^{5} \mathrm{SM}$ Creighton, ${ }^{6} \mathrm{R}$ Viner, ${ }^{5} \mathrm{D}$ Hodes. ${ }^{1}$ Department of Paediatrics, Whittington Hospital NHS Trust, London, UK; ${ }^{2}$ Research and Policy Division, Royal College of Paediatrics and Child Health, London, UK; ${ }^{3}$ Dept of Paediatrics, Birmingham Women and Children's Hospital NHS Foundation Trust, Birmingham, UK; ${ }^{4}$ British Paediatric Surveillance Unit, Royal College of Paediatrics and Child Health, London, UK; ${ }^{5}$ Department of Paediatrics, University College London Hospitals NHS Foundation Trust, London, UK; ${ }^{6}$ Institute of Child Health, University College London, London UK

\subsection{6/archdischild-2018-rcpch.48}

Aims Female genital mutilation (FGM) is the name given to procedures that involve partial or total removal or other injury to the female genitalia for nonmedical reasons. This study describes the presentation, incidence and clinical management of children with FGM in the UK and Republic of Ireland (ROI).

Methods Cases of FGM were reported using the established national British Paediatric Surveillance Unit (BPSU) reporting system. The data period is from November 2015-November 2017 with a 12 month follow up.

Results These interim results are from 120 cases reported (November 2015-September 2017). 61 (51\%) had confirmed FGM, 18 cases were reported in error or were duplicates, 36 questionnaires were incomplete [5 did not meet case definition]. $48 \%(n=29)$ of the 61 confirmed cases were classified as type 2 .

In over $72 \%$ of the 61 cases, the parent disclosed child's history of FGM. At the time of diagnosis, $80 \%$ of children $(n=49)$ were four years or older (11 cases not recorded). Most children were diagnosed between 5 years and 10 years 11 months $(n=27)$ or 11 years and 15 years 11 months $(n=20)$ with 3 cases diagnosed between 0 and 4 years 11 months. In $51 \%$ of cases FGM was said to have been performed when the child was between 0 and 3 years $(n=31)$. $93 \%(n=57)$ were performed before arrival to the UK.

$13 \%(n=8)$ of children had medical symptoms attributed to FGM, with $7 \%(n=4)$ of children identified to have mental health symptoms relating to FGM. No children presented with a history of labiaplasty or genital piercing.

Conclusion Numbers reported were lower than expected for UK estimated prevalence with fewer physical and mental health symptoms than anticipated. Further information is needed to determine illegality under UK law. These findings should be used to educate health, social care, police and education on prevention programmes to influence national policies.

Funding Department of Health England. 
114 MONITORING FOR HYPOGLYCAEMIC NEWBORNS SHOULD WE EXPAND OUR RISK CATEGORIES?

${ }^{1} \mathrm{M}$ Flavin, ${ }^{2} \mathrm{H}$ Osiovich, ${ }^{3} \mathrm{~K}$ Coughlin, ${ }^{4} \mathrm{~J}$ Ray, ${ }^{5} \mathrm{~L} \mathrm{Hu}$, ${ }^{6} \mathrm{~J}$ Andres Leon, ${ }^{4} \mathrm{M}$ Sgro, ${ }^{1} \mathrm{~A}$ Gallipoli, ${ }^{1} \mathrm{~K}$ Gregoire, 'L Barr, ${ }^{1} \mathrm{~K}$ Grewal. 'Paediatrics, Queen's University, Kingston, Canada; ${ }^{2} B C$ Children's Hospital, Vancouver, Canada; ${ }^{3}$ London Health Sciences Centre, London, Canada; ${ }^{4}$ St Michael's Hospital, Toronto, Canada; ${ }^{5}$ Children's Hospital Fudan, Shanghai, China;

${ }^{6}$ Public Health Agency of Canada, Ottawa, Canada

\subsection{6/archdischild-2018-rcpch.482}

Background and objectives Universal hypoglycaemia monitoring of newborns is not recommended. We wished to determine the incidence, presentation and case characteristics of hypoglycaemic newborns that were not being monitored.

Methods Through the Canadian Paediatric Surveillance Program we conducted a national study of severe hypoglycaemia in apparently low-risk full-term newborns. Inclusion criteria were: an otherwise healthy infant less than 96 hours old; gestational age 37-42 weeks; birth weight 2500-3999 grams; whole blood or serum glucose less than $2.0 \mathrm{mmol} / \mathrm{L}$ and IV dextrose used to treat the hypoglycaemia. Data were managed and analysed using IBM SPSS Statistics for Windows, Version 24.0 (Armonk, NY: IBM Corp.).

Results From April 2014 to March 2016, 177 cases were reported. There were 5 duplicates, 33 cases did not meet inclusion criteria and 46 questionnaires were not returned, leaving 93 confirmed cases. The estimated incidence was 1 in 8378 births. All cases were singletons, 56\% were first-borns and $65 \%$ were male. An $8 \%$ rate of First Nations cases was 3 -fold the population rate. Maternal hypertension was present in 23\%, 4-fold the overall pregnancy rate. Maternal obesity was double the overall pregnancy rate at 23\%. Concerning signs or feeding issues were present at diagnosis in $98 \%$. Median time to diagnosis was 4.1 hours. Mean blood glucose was $1.4 \pm 0.5$ hours (SD). Seventy eight percent had at least one of 4 potential perinatal stress indicators (emergency Caesarean Section, meconium at delivery, requiring resuscitation or cord artery $\mathrm{pH}<7.10)$. Those cases were more likely to be diagnosed before 6 hours $(p=0.03)$. Twenty five percent (23 cases) were small for gestational age (SGA) with birth weight $<10$ th centile, of which 5 had seizures and 5 had hyperinsulinism. Presentation with major clinical signs (seizure, apnoea or cyanosis) occurred in 20\%. Neurodevelopmental concern was present in $20 \%$ of all cases. Amongst 13 cases which had brain MRI, 11 were abnormal.

Conclusion While acknowledging the study's limitations, the impact of First Nations origin, maternal obesity, maternal hypertension and perinatal stress indicators warrant further study and possible incorporation into glucose monitoring guidelines. The data further supports adoption of norm-based standards to identify and monitor all SGA infants.

Funding Supported by grants from Queen's University Faculty of Health Sciences and the Public Health Agency of Canada.

\section{TEN YEARS OF ALCOHOL INTOXICATIONS IN ADOLESCENTS AND TREATMENT IN PAEDIATRIC DEPARTMENTS IN DUTCH HOSPITALS}

${ }^{1} \mathrm{~N}$ Van Der Lely, ${ }^{2} \mathrm{JJV}$ Hoof, ${ }^{1} \mathrm{I}$ Wolberink, ${ }^{1} \mathrm{~K}$ Nienhuis. ${ }^{1}$ Reinier de Graaf Gasthuis, Delft, The Netherlands; ${ }^{2}$ Behavioral Sciences Faculty, University of Twente, Enschede, The Netherlands
Aim Alcohol intoxication in children and adolescents is a severe health concern in current paediatrics. In this longitudinal study we monitored intake and treatment of 5893 adolescents in Departments of Paediatrics in Dutch hospitals over the years 2007 to 2016 .

Methods from 2007 till 2016 we collected data on all adolescents (inclusion criteria: aged younger than 18 and with a positive blood alcohol concentration (BAC), treated by a paediatrician in all Dutch hospitals. Within the Dutch Paediatric Surveillance System (NSCK), all paediatricians report adolescents and fill in a questionnaire, making use of a patient interview.

Results in total 5893 adolescents were treated, mainly $(4,678$; $88 \%)$ related to severe alcohol intoxication; mean age was 15.4 years, and 52\% were boys. BAC level increased during this period (1.82 in 2007 and 2.01 in 2016), and reduced consciousness lasted from 2.24 hours in 2007 till 3.12 hours in 2016). 11.4\% Of the adolescents with alcohol intoxication had simultaneous drug usage. The attitude of the parents changed during the years: in 2011 (first year of registration) $68 \%$ of the parents gave permission to their child to drink alcohol, in 2016 this decreased to only $19 \%$.

Conclusions alcohol intoxication treatment remains an issue of importance. This dataset enables us to conduct longitudinal and interesting analyses on alcohol intoxication characteristics in youngsters, medical treatment, and events leading up to the intoxication.

\section{I16 NUTRITIONAL RICKETS PRESENTING TO SECONDARY CARE IN CHILDREN (<16 YEARS) - A UK SURVEILLANCE STUDY}

${ }^{1} \mathrm{P}$ Julies, ${ }^{2} \mathrm{RM}$ Lynn, ${ }^{2} \mathrm{~K}$ Pall, ${ }^{2} \mathrm{M}$ Leoni, ${ }^{3} \mathrm{~A}$ Calder, ${ }^{4} \mathrm{Z}$ Mughal, ${ }^{5} \mathrm{~N}$ Shaw, ${ }^{6} \mathrm{H}$ McDevitt, ${ }^{7} \mathrm{C}$ MacDonnell, ${ }^{8} \mathrm{M}$ Blair. ${ }^{1}$ Royal Free Hospital, London, UK; ${ }^{2}$ BPSU, London, UK; ${ }^{3}$ Great Ormond Street Hospital, London, UK; ${ }^{4}$ Manchester University Hospital, Manchester, UK; ${ }^{5}$ Birmingham Children's Hospital, Birmingham, UK; ${ }^{6}$ University of Glasgow, Glasgow, UK; ${ }^{7}$ National Children's Hospital, Tallaght, Republic of Ireland; ${ }^{8}$ Imperial College, London, UK

\subsection{6/archdischild-2018-rcpch.484}

Aims Rickets is a disease of growing children with serious short and long-term complications. Although the prevalence of rickets has been reported widely to be increasing the actual national incidence of nutritional rickets (NR) in the United Kingdom (UK) is unknown. This study aims to describe the incidence, presentation, and clinical management of children with NR in the UK and ROI.

Methods Data was collected prospectively monthly between March 2015-March 2017 from 3500 paediatricians using British Paediatric Surveillance Unit reporting methodology with the following definition (table 1):

Abstract I16 Table 1
Clinical rickets with any of the following:
- Leg deformity/Swollen wrists or knees or ribs AND 250 hour vitamin $D<25 \mathrm{nmol} / \mathrm{L}$ with
one or more abnormalities of serum calcium, alkaline phosphatase, phosphate,
parathyroid hormone
OR
Radiological rickets with:
- Widening, cupping, splaying of metaphysis (of any long bone) AND 250HVitamin
D<25 nmol/L

